\title{
A dermoscopic study of nail involvement in chronic plaque psoriasis at a tertiary care center
}

\author{
Neikhrietsonuo Kesiezie ${ }^{1}$, Meriya Zacharia ${ }^{2}$, Aswini $\mathbf{R}^{3}$ \\ ${ }^{1}$ Senior Resident, ${ }^{2,3}$ Assistant Professor, Department of Dermatology, Venereology and Leprosy, Government Medical \\ College and Hospital, Kottayam, Kerala, India
}

Background: Psoriasis is a chronic inflammatory disease that affects millions of people throughout the world. Nail involvement is a common feature in psoriasis which is often overlooked despite causing significant functional impairment, disfigurement, and psychological stress to the patient. Dermoscopy is a non-invasive novel tool that enables a rapid and magnified visualization of the nail changes which are often imperceptible to the unaided eye. Aims and Objectives: To study the dermoscopic profile of nail changes in patients with chronic plaque psoriasis. Materials and Methods: The study was conducted from December 2018 to May 2020, in the Department of Dermatology of a tertiary care center in Kerala, including 155 patients of clinically diagnosed chronic plaque psoriasis who had nail involvement. A detailed history was noted along with a clinical and dermoscopic examination of all 20 nails of each patient. Results: The most common finding on dermoscopic examination of the nails was onycholysis seen in $91 \%$, followed by pitting $(78.8 \%)$ and subungual hyperkeratosis $(78.1 \%)$. Other findings noted in our study which are not commonly described are wavy lamellar microsplitting $(41.9 \%)$, cuticular hypertrophy $(18.1 \%)$, pseudofiber sign $(16.1 \%)$, dilated nailfold capillaries $(12.3 \%)$, capillary prominence at onychodermal band $(7.7 \%)$, dilated hyponychial capillaries $(7.7 \%)$, and fuzzy lunula $(7.1 \%)$. Conclusion: Nails are frequently involved in psoriasis but often overlooked. Dermoscope is an effective tool that facilitates the assessment of nail changes in psoriasis.
Access this article online

\section{Website:}

http://nepjol.info/index.php/AJMS

DOI: 10.3126/ajms.v13i3.40878

E-ISSN: 2091-0576

P-ISSN: 2467-9100

Copyright (c) 2022 Asian Journal of Medical Sciences

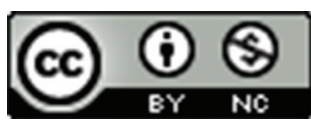

This work is licensed under a Creative Commons Attribution-NonCommercial 4.0 International License.

Key words: Dermoscopy; Nail; Psoriasis

\section{INTRODUCTION}

Psoriasis is a chronic inflammatory and proliferative condition of the skin characterized by red, scaly, sharply demarcated and indurated plaques affecting mainly the extensor surfaces of limbs and scalp. ${ }^{1}$ Nail involvement occurs in about $15-50 \%$ of cases of psoriasis. ${ }^{2}$ Isolated nail changes without any skin involvement are seen in approximately $5 \%$ of psoriasis patients. ${ }^{3}$ Nail psoriasis results from the involvement of the nail matrix or the nail bed by the psoriatic pathology, each of which is characterized by distinct clinical presentations. ${ }^{4}$ Pitting, red spots in lunula, crumbling and leukonychia are features of nail matrix involvement, whereas nail bed involvement presents as onycholysis, subungual hyperkeratosis, splinter hemorrhages, and oil drop sign/salmon patch. ${ }^{4}$

Dermoscopy is a valuable diagnostic modality that allows a better visualization of the alterations which are not easily visible. Dermoscopy was initially used for the diagnosis of melanoma, however, in recent years it has been increasingly recognized as an important diagnostic tool in many skin conditions including nail disorders which is termed as onychoscopy. ${ }^{5}$ The nail changes in psoriasis are often detected on gross examination, but in few cases, dermoscopy of the nail can aid in the detection of subtle changes especially in subclinical nail involvement. ${ }^{6}$ This study was done with the primary 
objective of describing the dermoscopic features in the psoriatic nail.

\section{Aims and objectives}

1. To describe the dermoscopic features in nail psoriasis

2. To determine the frequency of each finding.

\section{MATERIALS AND METHODS}

The study was a descriptive cross-sectional study conducted at the Dermatology outpatient department, Government Medical College and Hospital, Kottayam, Kerala over a period of 18 months from December 2018 to May 2020. The study was approved by the Institutional Review Board Government Medical College Kottayam (IRB:112/2018) and written consents were obtained from all patients prior to enrolling in the study. A total of 155 patients who were clinically diagnosed as chronic plaque psoriasis with nail involvement were included in the study. All the other forms of psoriasis and patients with isolated nail involvement were excluded. Informed consent and a detailed history and clinical examination were done. Dermoscopic examination of all twenty nails of each patient was done using a handheld dermoscope (Dermlite DL3N) equipped with a camera. The part of the nail examined included nail plate, nail bed, proximal and lateral nailfolds, cuticle, and hyponychium. All the dermoscopic images were evaluated by all the authors for the presence of common features such as pitting, red spots in lunula, leukonychia, crumbling, onycholysis, subungual hyperkeratosis, splinter hemorrhages, and oil drop sign. The presence of any other features was also looked for and recorded.

\section{Statistical analysis}

The data obtained in the study were coded, verified, and entered in Microsoft Excel and was statistically analyzed using statistical package for social sciences software. Dermoscopic features were all described using percentages.

\section{RESULTS}

A total of 155 patients who were clinically diagnosed as chronic plaque psoriasis with nail involvement were included in this study, out of which there were $115(74.2 \%)$ males and $40(25.8 \%)$ females with male: female ratio of $2.9: 1$. The mean age of the study population was $52.75 \pm 13.64$ years. The mean age of onset of nail changes was $47.77 \pm 14.11$ years. The mean duration of nail psoriasis was $5.30 \pm 6.70$ years. In this study, $56.8 \%(n=88)$ of the patients had a history of cutaneous psoriasis prior to the onset of nail changes, 10.3\% $(n=16)$ had a history of nail changes prior to the onset of cutaneous psoriasis and $32.9 \%(\mathrm{n}=55)$ of patients had simultaneous onset of cutaneous psoriasis and nail changes. On dermoscopic examination of the nails, it was observed that the majority of the patients $(54.19 \%, \mathrm{n}=84)$ had more number of fingernails involved than toenails. Of the various findings on dermoscopy, onycholysis (Figure 1) was the most common finding seen in $91 \%(\mathrm{n}=141)$ followed by pitting in $78.7 \%(\mathrm{n}=122)$ and subungual hyperkeratosis in $78.1 \%$ (121) of patients. Other features noted in order of decreasing frequency are splinter haemorrhages $(63.9 \%, \mathrm{n}=99)$, leukonychia $(61.9 \%, \mathrm{n}=96)$, longitudinal ridges $(49.7 \%, \mathrm{n}=77)$, thickening of the nail plate $(41.9 \%$, $\mathrm{n}=65)$, Beau's line $(34.8 \%, \mathrm{n}=54)$, oil drop sign/salmon patch $(29.6 \%, \mathrm{n}=46)$, dystrophy/crumbling $(29 \%, \mathrm{n}=45)$, longitudinal melanonychia $(20 \%, \mathrm{n}=31)$, and red spots in lunula $(11.6 \%, \mathrm{n}=18)$ (Table 1).

Some additional findings (Figure 2) noted solely on dermoscopic examination in decreasing order are wavy lamellar microsplitting $(41.9 \%, \mathrm{n}=65)$ (Figure 3), cuticular hypertrophy (18.1, $\mathrm{n}=28$ ), Pseudo fiber sign $(16.1 \%, n=25)$ (Figure 4), dilated proximal nail fold capillaries $(12.3 \%, \mathrm{n}=19)$, capillary prominence at the onychodermal band $(7.7 \%, \mathrm{n}=12)$ (Figure 5), dilated hyponychial capillaries $(7.7 \%, \mathrm{n}=12)$ and fuzzy lunula $(7.1 \%, \mathrm{n}=11)$ (Figure 6).

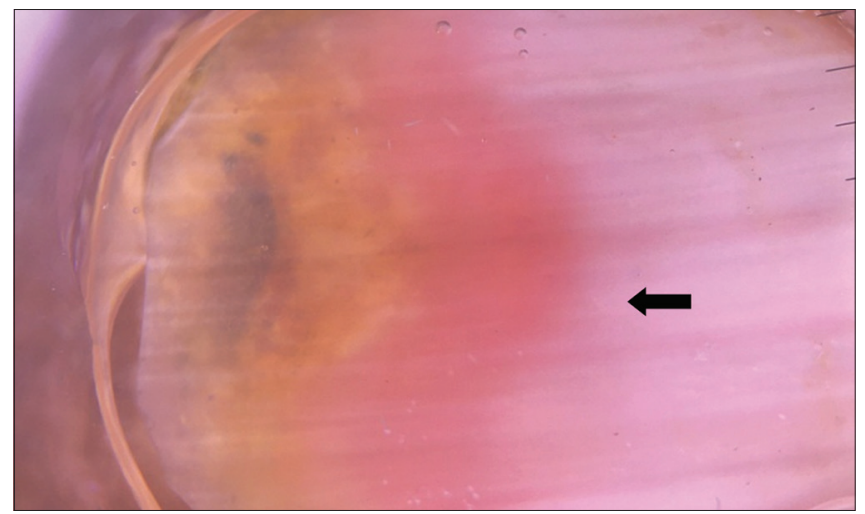

Figure 1: Dermoscopy of nail showing distal onycholysis with an erythematous border (black arrow)

\begin{tabular}{lcc}
$\begin{array}{l}\text { Table 1: Distribution of dermoscopic nail } \\
\text { findings }\end{array}$ \\
\hline Findings & Frequency (n) & Percentage \\
\hline Pitting & 122 & 78.7 \\
Red spots in lunula & 18 & 11.6 \\
Dystrophy & 45 & 29.0 \\
Leukonychia & 96 & 61.9 \\
Onycholysis & 141 & 91.0 \\
Subungal hyperkeratosis & 121 & 78.1 \\
Salmon patch & 46 & 29.6 \\
Splinter hemorrhage & 99 & 63.9 \\
Thickening of nails & 65 & 41.9 \\
Longitudinal ridges & 77 & 49.7 \\
Beaus lines & 54 & 34.8 \\
Longitudinal melanonychia & 31 & 20 \\
\hline
\end{tabular}




\section{DISCUSSION}

Nail psoriasis presents with varied clinical signs depending upon the structure of the nail involved. Most of the features in nail psoriasis are non-specific and can be detected on gross examination. However, dermoscopy is useful in detecting subtle changes and can also aid in deciding the treatment options for the patient based upon the structure of the nail affected. ${ }^{7}$

\section{Pitting}

Pitting refers to the depressions on the nail plate which occur due to parakeratosis of the proximal nail matrix. As the nail grows these parakeratotic cells are sloughed off leaving deep and coarse pits on the nail plate. ${ }^{8} \mathrm{~A}$ diagnosis of psoriasis can be considered in a patient with more than 20 fingernail pits. ${ }^{8}$ In our study, we observed pitting as the second most common dermoscopic finding which was noted in $78.7 \%$ of the patients. However Yadav and Khopkar $^{6}$ and Wanniang et al., ${ }^{9}$ determined pitting as the most common dermoscopic finding in their studies seen in $39.13 \%$ and $84 \%$, respectively. In another study, Polat and Kapicioglu, ${ }^{4}$ observed pitting to be the third most

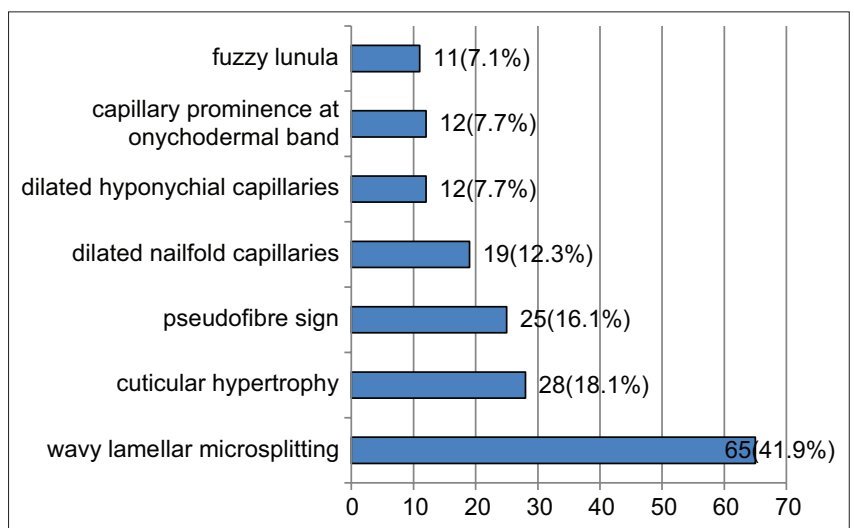

Figure 2: Additional dermoscopic findings of nails

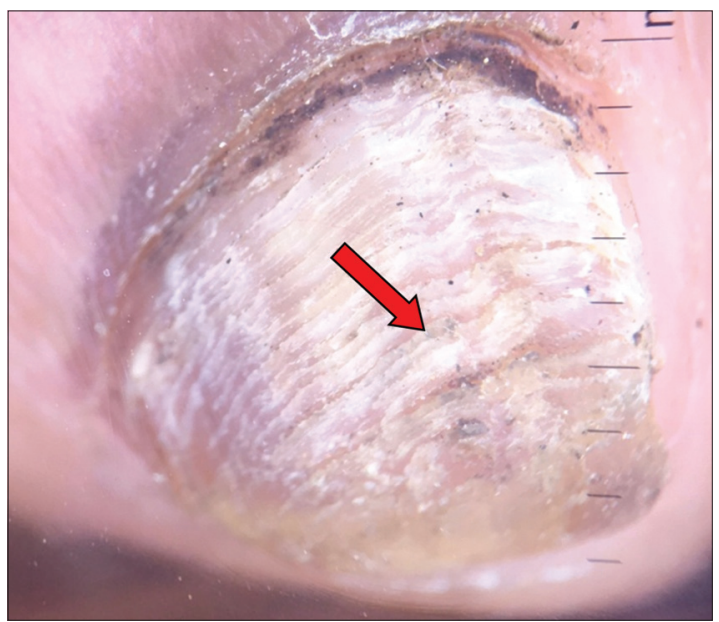

Figure 3: Dermoscopy of wavy lamellar microsplitting (red arrow) common dermoscopic finding which was noted in $77.5 \%$ of their patients.

\section{Onycholysis}

Onycholysis refers to the detachment of the nail plate from the nail bed. ${ }^{8}$ Yorulmaz and Artuz, ${ }^{10}$ described a linear erythematous border around the onycholysis, which they

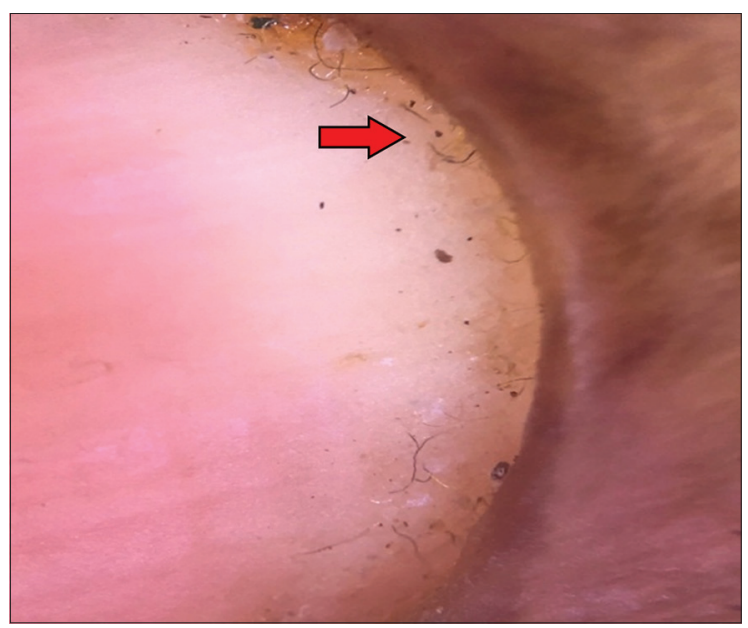

Figure 4: Pseudo-fiber sign (red arrow) appearing as black filamentous structures along the cuticle on dermoscopic examination

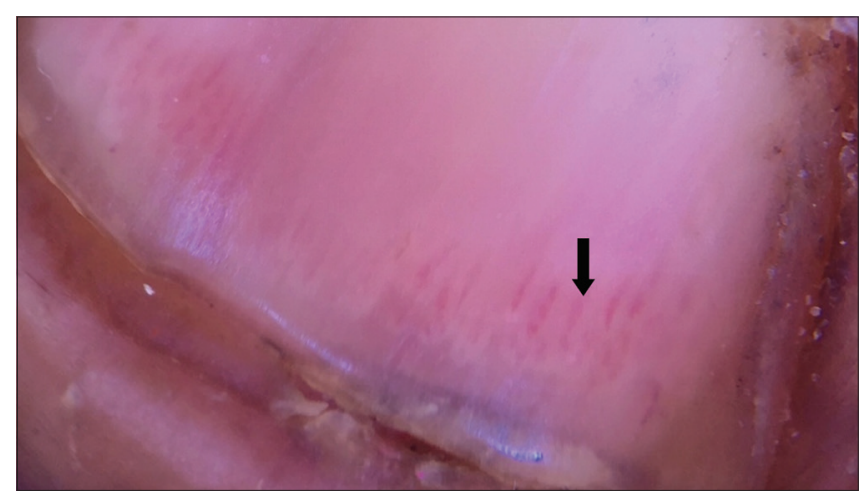

Figure 5: Dermoscopy showing dilated capillaries with surrounding prominent clear halo seen over the onychodermal band (black arrow) on the nail plate

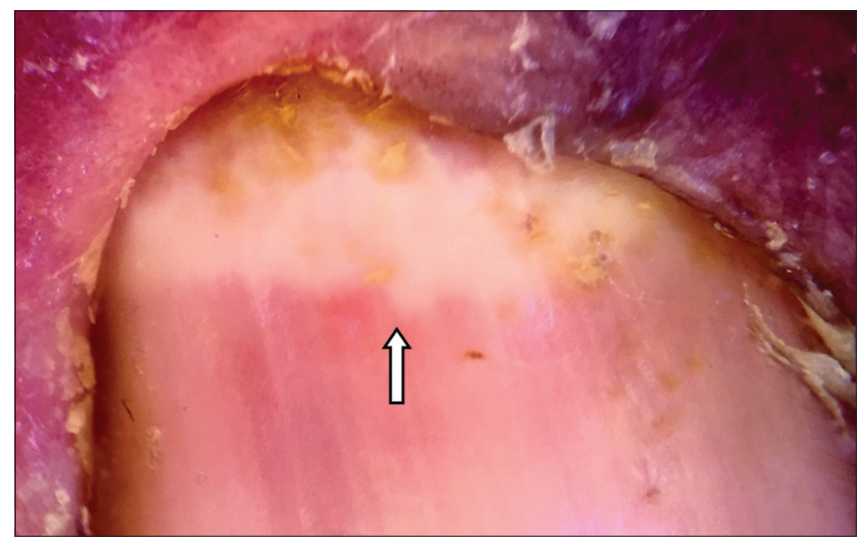

Figure 6: Dermoscopy of fuzzy lunula (white arrow) 
considered to be specific for psoriatic nails and thus aids in differentiating onycholysis due to psoriasis from other causes. We observed onycholysis with an erythematous border as the most common finding in our dermoscopic study noted in $91 \%$ of our study subjects. Studies by Yadav and Khopkar, ${ }^{6}$ and Wanniang et al., ${ }^{9}$ reported onycholysis as the second most common dermoscopic findings seen in $21.7 \%$ and $54 \%$ of their patients, respectively.

\section{Salmon patch}

Salmon patch is considered a specific diagnostic sign for nail psoriasis which can be seen as translucent red yellow patch on the nail plate that appears like a drop of oil under the nail plate, hence also known as oil drop sign. ${ }^{11}$ Salmon patch results from the focal nail bed parakeratosis leading to focal onycholysis and entrapment of serum and cellular debris within the areas of onycholysis. ${ }^{11}$ On dermoscopic examination we observed salmon patch in $29.6 \%$ of our patients. Wanniang et al., ${ }^{9}$ observed salmon patch in $44 \%$ of the patients while Yadav and Khopkar, ${ }^{6}$ observed this finding in only $4 \%$ of their study population.

\section{Subungual hyperkeratosis}

Subungual hyperkeratosis results from raising of the nail plate off the nail bed due to the deposits of cells that have not undergone desquamation. ${ }^{2}$ On dermoscopic examination, $78.1 \%$ of our patients had subungual hyperkeratosis. Subungual hyperkeratosis was the most common nail bed finding in a dermoscopic study by Chauhan et al., which was noted in fingernails of $52.8 \%$ of patients and in toenails of $85.14 \%$ of patients. ${ }^{12}$

\section{Leukonychia}

Leukonychia results from the psoriasis induced parakeratosis affecting the intermediate and ventral matrices which form the undersurface of the nail plate and due to internal desquamation of parakeratotic cells, the affected areas appear white. ${ }^{8}$ In our study, we observed leukonychia in $61.9 \%$ of our patients. Polat and Kapicioglu, ${ }^{4}$ noted that on dermoscopy of psoriatic nails leukonychia was present in $92.5 \%$ of the patients while Wanniang et al., ${ }^{9}$ reported leukonychia in only $22 \%$ of their patients.

\section{Splinter hemorrhages}

Splinter hemorrhages occur due to extravasation of blood along the grooves beneath the nail plate when dilated capillaries in the nail bed rupture and they appear as reddish-brown to purplish-black striae in a longitudinal distribution on nail plate. ${ }^{9}$ In our present study, we observed splinter hemorrhages in $63.9 \%$ of our patients. Splinter hemorrhages were reported in $62 \%$ and $80 \%$ of patients in dermoscopic studies conducted by Wanniang et al., ${ }^{9}$ and Polat and Kapicioglu, ${ }^{4}$ respectively.

\section{Red spots in lunula}

Erythematous spots on lunula occur as result of the involvement of the intermediate and ventral matrices. ${ }^{2}$ We reported this finding in $11.6 \%$ of our patients on dermoscopic examination. Polat and Kapicioglu, ${ }^{4}$ and Chauhan et al., ${ }^{12}$ also observed red spots in lunula in $5 \%$ and $10 \%$ of their patients, respectively.

\section{Crumbling of nails}

When the entire nail matrix is involved in severe psoriasis the plate becomes dystrophic and assumes a crumbly appearance. $^{2}$ We observed crumbling of nails in $29 \%$ of our patients in our present dermoscopic study. In other studies by Yorulmaz and Artuz, ${ }^{10}$ and Wanniang et al., nail crumbling was reported in $6 \%$ and $17.9 \%$ of psoriasis cases respectively.

\section{Pseudo-fiber sign}

Pseudo-fiber sign was first described by Yorulmaz and Artuz, ${ }^{10}$ who observed this finding in $34.3 \%$ of their psoriatic patients on dermoscopic examination. Pseudofiber sign is described as filamentous structures which are red and black in color, located in proximity to the cuticle or under the hyponychium and it is suggested to be the representative of bare capillaries related to nail bed psoriasis. ${ }^{12}$ In our present dermoscopic study, we reported this finding in $16.1 \%(n=25)$ of our patients. The other dermoscopic study which reported pseudo-fiber sign is Wannaing et al., in which $18 \%$ of their psoriatic patients had this feature on dermoscopic examination.

\section{Wavy lamellar microspliting}

Wavy lamellar microspliting appeared as multiple transverse wavy bands of lamellar splitting on the nail plate on dermoscopic examination of the psoriatic nails. This feature was observed in $41.9 \% \quad(n=65)$ of our psoriatic patients and has not been described previously in literature in association with nail psoriasis although a similar feature has been described by Kayarkatte et al., ${ }^{13}$ in association with total dystrophic onychomycosis. As our study was a descriptive study we were unable to evaluate if wavy lamellar microsplitting was specifically caused by psoriasis in our patients.

\section{Fuzzy lunula}

Fuzzy lunula is another newly reported feature of nail psoriasis first described by Chauhan et al., ${ }^{12}$ as irregular wide white lunula which was observed in $30.90 \%$ of their patients. We also noted this appearance of fuzzy lunula in $7 \%(n=11)$ of our patients.

\section{Dilated nail fold capillaries}

In our present study, we noted the presence of dilated capillaries in the proximal nail fold on dermoscopic 
examination in $12.3 \%$ of patients. A study by Chauhan et al., ${ }^{12}$ also reported dilated nailfold capillaries in proximal nailfold in $5.8 \%$ and $0.9 \%$ of fingernails and toenails respectively. Zaric et al., ${ }^{14}$ and Bushan et al., ${ }^{15}$ both reported a reduction in the size of capillaries of proximal nailfold in psoriatic patients with psoriatic arthritis. Bushan et al., ${ }^{15}$ also found a reduction in the capillary density in proximal nail fold of patients with nail psoriasis or psoriasis with nail and joint involvement.

\section{Dilated capillaries in hyponychium}

Dilated capillaries observed in the hyponychium could be due to the underlying changes in the dermal vasculature and were observed in 7.7\% $(\mathrm{n}=12)$ of patients in our dermoscopic study. Dermoscopy of hyponychium on clinically involved nails showed dilated tortuous and irregularly arranged capillaries in fingernails of $2.9 \%$ of patients and in toenails of $0.9 \%$ of patients in a study conducted by Chauhan et al., ${ }^{12}$ Wanniang et al., ${ }^{9}$ reported this finding in $10 \%$ of their cases.

\section{Prominent capillaries at the onychodermal band}

Yadav and Khopkar, ${ }^{6}$ described bright red to dusky colored dilated vessels arranged in parallel over the onychodermal band on the nail plate which appeared as fusiform dilatation surrounded by a prominent halo in $13.23 \%$ of their patients. We also noted similar findings in $7.7 \%(\mathrm{n}=12)$ of patients in our dermoscopic study.

\section{Cuticular hypertrophy}

Hypertrophy of the cuticle was seen in $18.1 \%(n=28)$ of the psoriatic patients enrolled in our study. Other studies reporting cuticular hypertrophy in psoriatic nails include Wanniang et al., ${ }^{9}$ in $22 \%$ of patients and Polat and Kapicioglu in $2.5 \%$ of their patients.

\section{Other findings}

Other non-specific findings which were observed in our dermoscopic study of psoriatic nails include thickening of nail plate $(38.7 \%)$, Beaus lines $(34.8 \%)$, longitudinal melanonychia (20\%), and longitudinal ridges $(49.70 \%)$.

\section{Limitations of the study}

1. Small sample size.

2. Standard handheld dermoscope with magnification of $10 \mathrm{X}$ only which makes visualization of vascular structures difficult.

\section{CONCLUSION}

Our study comprehensively describes the dermoscopic features of nail involvement in chronic plaque psoriasis. We observed onycholysis to be the most common dermoscopic finding in our study which contrasted other studies where pitting is often reported as the most common finding associated with nail psoriasis. We also observed some findings which are less reported in literature such as wavy lamellar micro splitting, cuticular hypertrophy, pseudo fiber sign, dilated nail fold capillaries, capillary prominence at the onychodermal band, dilated hyponychial capillaries, and fuzzy lunula. Wavy lamellar micro splitting of nail plate has not been previously described in the literature in association with nail psoriasis but further comparative studies may be needed to clearly describe its association with psoriasis as our study is a descriptive study. We would like to highlight the importance of careful evaluation of nails in a psoriatic patients and the utility of a dermoscope as a bridge between clinical and histopathological examinations obviating the need of invasive diagnostic procedures like biopsy.

\section{ACKNOWLEDGMENT}

We acknowledge the contribution of the staff members and the patients attending the Dermatology OPD for their valuable help and cooperation during the period of the study.

\section{REFERENCES}

1. Burden $A D$ and Kirby B. Psoriasis and Related disorders. In: Griffith CE, Barker J, Bleiker T, Chalmers R and Creamer D, editors. Rook's Textbook of Dermatology. $9^{\text {th }}$ ed., Vol. 2. Oxford: Wiley Blackwell; 2016. p. 35-48.

2. Jiaravuthisan MM, Sasseville D, Vender RB, Murphy $F$ and Muhn CY. Psoriasis of the nail: Anatomy, pathology, clinical presentation, and a review of the literature on therapy. J Am Acad Dermatol. 2001;57(1):1-27.

https://doi.org/10.1016/j.jaad.2005.07.073

3. Zalaudek I, Argenziano G, Stefani AD, Ferrara G, Marghoob AA, Hofmann-Wellenhof $R$, et al. Dermoscopy in general dermatology. Dermatology. 2006;212(1):7-18. https://doi.org/10.1159/000089015

4. Polat A and Kapicioglu Y. Dermoscopic findings of psoriatic nail and their relationship with disease severity. Turk Arch Dermatol Venerol. 2017;51(4):119-123.

http://dx.doi.org/10.4274/turkderm.54289

5. Errichetti $E$ and Stinco G. Dermoscopy in general dermatology: A practical overview. Dermatol Ther. 2016;6(4):471-507.

https://dx.doi.org/10.1007\%2Fs13555-016-0141-6

6. Yadav TA and Khopkar US. Dermoscopy to detect signs of subclinical nail involvement in chronic plaque psoriasis: A study of 68 patients. Indian J Dermatol. 2015;60(3):272-275. https://dx.doi.org/10.4103\% 2F0019-5154.156377

7. Pasch MC. Nail psoriasis: A review of treatment options. Drugs. 2016;76(6):675-705.

https://doi.org/10.1007/s40265-016-0564-5

8. Dogra A and Arora AK. Nail psoriasis: The journey so far. Indian J Dermatol. 2014;59(4):319-333.

https://doi.org/10.4103/0019-5154.135470 
9. Wanniang N, Navya A, Pai V and Ghodge R. Comparative study of clinical and dermoscopic features in nail psoriasis. Indian Dermatol Online J. 2020;11(1):35-40.

https://dx.doi.org/10.4103\% 2Fidoj.IDOJ_51_19

10. Yorulmaz A and Artuz F. A study of dermoscopic features of nail psoriasis. Postepy Dermatol Alergol. 2017;34(1):28-35. https://dx.doi.org/10.5114\%2Fada.2017.65618

11. Kouskoukis CE, Scher RK and Ackerman AB. The "oil drop" sign of psoriatic nails: A clinical finding specific for psoriasis. Am J Dermatopathol. 1983;5(3):259-262.

https://doi.org/10.1097/00000372-198306000-00012

12. Chauhan A, Singal A, Grover C and Sharma S. Dermoscopic features of nail psoriasis: An observational, analytical study. Skin
Appendage Disord. 2020;6(4):207-215.

https://doi.org/10.1159/000508165

13. Kayarkatte $M$, Singal $A$, Pandhi D, Das $S$ and Sharma $S$. Nail dermoscopy findings in the diagnosis of primary onychomycosis: A cross sectional study. Indian J Dermatol. 2020;86(4):341-349. https://doi.org/10.4103/ijdv I.ijdvl_100_19

14. Zaric D, Clemmensen OJ, Worm AM and Stahl D. Capillary microscopy of the nailfold in patients with psoriasis and psoriatic arthritis. Dermatology. 1982;164(1):10-14. https://doi.org/10.1159/000250060

15. Bushan M, Moore T, Herric AL and Griffiths CE. Nailfold video capillaroscopy in psoriasis. BrJDermatol. 2000;142(6):1171-1176. https://doi.org/10.1046/j.1365-2133.2000.03544.x

\section{Authors' Contributions:}

NK- Contributed to the development of the protocol, conducted the research and data collection, statistical analysis and authored the article; MZ- Contributed to the development of protocol, intellectual content and authored the article; AR-Contributed to the development of protocol, intellectual content and authored the article

\section{Work attributted to:}

Government Medical College and Hospital, Kottayam - 686 008, Kerala, India

ORCID ID:

Dr. Neikhrietsonuo Kesiezie - (D) https://orcid.org/0000-0002-6022-5397

Dr. Meriya Zacharia - (1) https://orcid.org/0000-0002-0348-8887

Dr. Aswini R - (D) https://orcid.org/0000-0001-5822-8604 